\title{
Relationship of Change in Plasma Clozapine/N-desmethylclozapine Ratio with Cognitive Performance in Patients with Schizophrenia
}

\author{
Royun Park', Seoyoung Kim², and Euitae Kim ${ }^{2,3,4} \bowtie$ \\ ${ }^{1}$ Department of Neuropsychiatry, Seoul National University Hospital, Seoul, Republic of Korea \\ ${ }^{2}$ Department of Neuropsychiatry, Seoul National University Bundang Hospital, Seongnam, Republic of Korea \\ ${ }^{3}$ Department of Psychiatry, Seoul National University College of Medicine, Seoul, Republic of Korea \\ ${ }^{4}$ Department of Brain \& Cognitive Sciences, Seoul National University College of Natural Sciences, Seoul, Republic of Korea
}

Objective The clozapine/N-desmethylclozapine (NDMC) ratio is proposed to be used as a predictor of cognitive performance in clozapine-treated patients, as its principal metabolite, NDMC, has an opposite action with clozapine on the cholinergic system. The aim of this study is to determine whether clozapine has influence on cognitive performance in accordance with changes in the clozapine/ NDMC in patients with schizophrenia.

Methods The data of fifteen patients with schizophrenia, who had initial and follow-up assessments after starting clozapine treatment, were retrospectively collected. The assessments included clinical scale, cognitive battery, and pharmacological data including plasma concentrations of clozapine and NDMC. The data were analyzed with Pearson correlation and stepwise multiple regression analyses.

Results $\Delta$ Attention/vigilance, $\Delta$ social cognition, and $\Delta$ composite score had a significant correlation with $\Delta$ clozapine/NDMC ratio, while $\Delta$ Working memory had correlation with $\Delta$ clozapine concentration and $\Delta$ NDMC concentration, and $\Delta$ social cognition had association with $\Delta$ clozapine concentration. Multiple regression analysis showed that $\Delta$ attention/vigilance had negative association with $\Delta$ clozapine/NDMC ratio, $\Delta$ working memory had negative relation with $\Delta$ clozapine concentration, and that $\Delta$ social cognition had negative association with $\Delta$ clozapine concentration.

Conclusion This finding implicates that lowering the clozapine/NDMC ratio could enhance cognition in patients with schizophrenia treated with clozapine.

Psychiatry Investig 2020;17(11):1158-1165

Key Words Clozapine, Cognition, Attention, Schizophrenia.

\section{INTRODUCTION}

Cognitive and negative symptoms of schizophrenia tend to be chronic and are associated with poor functional outcomes, compared to positive symptoms of schizophrenia. ${ }^{1}$ Cognitive impairments in schizophrenia have been identified in a wide range of domains, including verbal memory, working memory, motor speed, attention, executive function, and verbal fluency. ${ }^{2}$ These impairments have been shown to affect functioning in everyday life, including interpersonal relationships and

\footnotetext{
Received: August 17, 2020 Revised: October 5, 2020

Accepted: October 5, 2020

$\triangle$ Correspondence: Euitae Kim, MD, PhD

Department of Neuropsychiatry, Seoul National University Bundang Hospital, 82 Gumi-ro 173beon-gil, Bundang-gu, Seongnam 13620, Republic of Korea

Tel: +82-31-787-7435, Fax: +82-31-787-4058, E-mail: euitae.kim@snu.ac.kr

(ac) This is an Open Access article distributed under the terms of the Creative Commons Attribution Non-Commercial License (https://creativecommons.org/licenses/bync/4.0) which permits unrestricted non-commercial use, distribution, and reproduction in any medium, provided the original work is properly cited.
}

work skills. ${ }^{3,4}$ Although more than 50 clinical trials have been conducted in patients with schizophrenia, no medications have yet been approved by the FDA to treat cognitive impairments in patients with schizophrenia.,

Clozapine is effective for treatment-resistant schizophrenia.? Notably, clozapine is more efficacious for positive and negative symptoms and for improving patients' quality of life in treatment-resistant schizophrenia, compared to first-generation antipsychotics. ${ }^{8}$ Nevertheless, the effect of clozapine on cognitive symptoms remains controversial. In the past, clozapine was presumed to produce pro-cognitive effects through its multireceptor action, which increased hippocampal acetylcholine efflux, ${ }^{9,10}$ however, clinical trials regarding the cognitive effects of clozapine in patients with schizophrenia have shown mixed results, ranging from deleterious ${ }^{11}$ to beneficial. ${ }^{6,12}$

The conflicting results regarding the effects of clozapine on cognition are presumably related in part to the differing actions of clozapine and its major active metabolite, N-desmethylclo- 
zapine (NDMC), on the cholinergic system. ${ }^{13,14}$ Clozapine is an antagonist of muscarinic M1, M3, and M5 receptors; thus, it has anti-cholinergic activity, ${ }^{15,16}$ which may worsen cognition. In contrast, NDMC is a potent partial agonist of those same receptors and is predicted to enhance cognition. ${ }^{17,18}$ Furthermore, clozapine metabolism significantly varies among patients, resulting in NDMC plasma levels ranging from $20 \%$ to $150 \%$ of clozapine concentrations. ${ }^{19}$ Thus, the anticholinergic burden and its consequent effects on cognitive function are likely to be more affected by the clozapine/NDMC ratio than by the clozapine dose or either concentration alone. ${ }^{20}$

For this reason, previous studies have examined the association between cognitive performance and the clozapine/NDMC ratio in patients treated with clozapine. For example, negative correlations have been reported between the clozapine/NDMC ratio and some cognitive domains, including working memory, attention, and executive functioning. ${ }^{20-23}$ However, all of the studies mentioned above were cross-sectional in design; thus, the impact of change in the clozapine/NDMC ratio on cognitive performance remains largely unknown.

To determine whether clozapine influenced improvement in cognitive performance in accordance with a change in clozapine/NDMC ratio ( $\triangle$ clozapine/NDMC ratio) in patients with schizophrenia, previous longitudinal study examined patients with treatment-resistant schizophrenia for whom data of two or more standardized cognitive assessments and clozapine/ NDMC ratio measurements were available within 12-week study period. ${ }^{24}$ However, that study had several limitations, including its analysis of patients with unknown clozapine treatment duration, use of simplified versions of clinical and cognitive assessments, and analysis of composite score alone (because of its limited sample size). Hence, we designed this study to address these limitations.

This study was performed to determine the association between improvement in cognitive performance and $\Delta$ clozapine/ NDMC ratio in patients with schizophrenia who started and continued clozapine treatment for more than 2 months. We hypothesized that the $\Delta$ clozapine/NDMC ratio would be negatively associated with improvement in cognitive function, and we designed the study to determine which cognitive domains were most strongly associated with the $\triangle$ clozapine/NDMC ratio.

\section{METHODS}

This study was approved by the Institutional Review Board of Seoul National University Bundang Hospital, Gyeonggi-do, Korea and was conducted in accordance with the Helsinki Declaration of 1975, as revised in 2008 (B-1910-570-103).

\section{Data acquisition}

Routine clinical data were retrospectively collected from patients who attended Seoul National University Bundang Hospital between January 2016 and September 2019. Patients were included if they 1 ) were diagnosed with schizophrenia or schizoaffective disorder, based on ICD-10 criteria; 2) were older than 19 years; and 3) started clozapine treatment and completed initial and follow-up assessments (e.g., pharmacological, clinical, and cognitive assessments). Initial assessments were defined as those performed within one month (30 days) of clozapine treatment initiation; follow-up assessments were defined as those performed more than 2 months (60 days) after initiation of clozapine (Figure 1).

\section{Demographic data and pharmacological assessments}

Age, sex, and years of education at the initial assessment were collected as demographic data. Participants underwent blood sampling as part of routine patient monitoring to detect the plasma levels of clozapine and NDMC, which were obtained through liquid chromatography-tandem mass spectrometry. ${ }^{25}$ Other pharmacological data, such as clozapine dose and other medications (e.g., antipsychotics, antidepressants, mood stabilizers, benzodiazepines, anticholinergics, and beta blockers), were obtained from patients' medical records.

\section{Clinical and cognitive assessments}

Clinical symptoms were assessed using the Positive and Negative Syndrome Scale (PANSS) ${ }^{26}$ Cognition was assessed

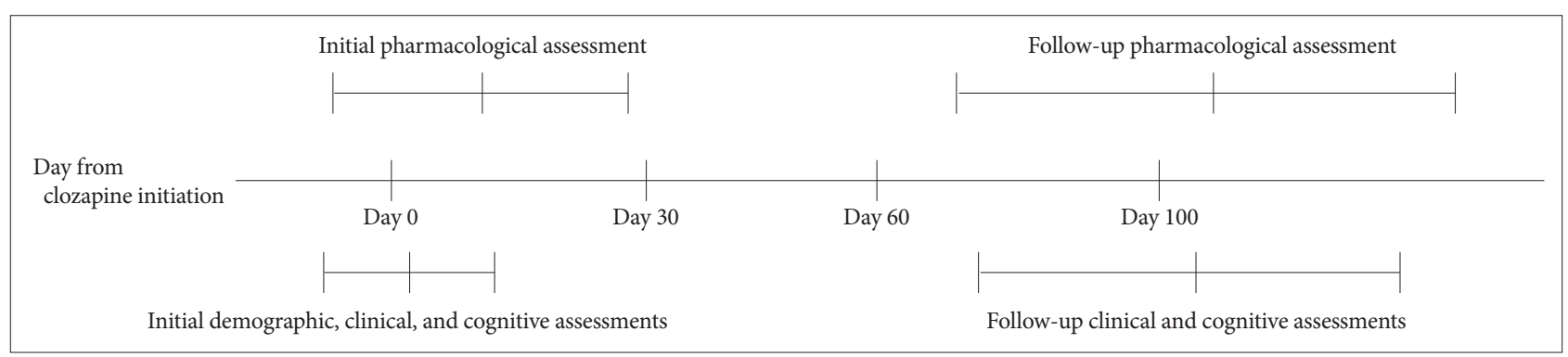

Figure 1. Schematic diagram of assessment schedule summarized from retrospectively obtained clinical data. The midline and error bars indicate mean and standard deviation of days after the initiation of clozapine treatment that each assessment was made. 
using the Measurement and Treatment Research to Improve Cognition in Schizophrenia (MATRICS) Consensus Cognitive Battery (MCCB), which was developed to assess cognitive treatment effects in clinical trials involving patients with schizophrenia; it is considered the Food and Drug Administration (FDA) gold standard outcome measure for that purpose. ${ }^{27,28}$ The MCCB includes 10 tests that assess the following seven cognitive domains:

1) Processing speed [Trail Making Test, part A (TMT), symbol coding task of the Brief Assessment of Cognition in Schizophrenia (BACS), and category fluency (Fluency)]

2) Attention/Vigilance [Continuous Performance Test-Identical Pairs (CPT-IP)]

3) Working memory [Spatial Span test (SS) and Letter-Number Span test (LNS) of the Wechsler Memory Scale-3rd Ed.]

4) Verbal learning [Hopkin's Verbal Learning Test-Revised (HVLT-R)]

5) Visual learning [Brief Visuospatial Memory Test-Revised (BVMT-R)]

6) Reasoning and problem solving [Mazes test (Mazes) of the Neuropsychological Assessment Battery]

7) Social cognition [Managing Emotions test (ME) of the Mayer-Salovey-Caruso Emotional Intelligence Test]

T-scores for each cognitive domain, standardized and corrected for age and sex, were used as individual outcome measures. ${ }^{29}$ The overall composite score was the standardized total of scores for the seven domains.

\section{Statistical analysis}

All statistical analyses $\left({ }^{*} \mathrm{p}<0.05\right.$ and $\left.{ }^{* *} \mathrm{p}<0.01\right)$ were performed using IBM SPSS Statistics, version 23.0 (IBM Corp., Armonk, NY, USA). To compare initial and follow-up assessments of pharmacological, clinical, and cognitive variables, paired t-tests or Wilcoxon signed-rank tests were used, in accordance with homogeneity and normality. Four patients underwent initial pharmacologic assessments before initiation of clozapine treatment, resulting in initial clozapine and NDMC concentration values of 0 ; the initial clozapine/NDMC ratios of these four patients were defined as 0 . Pearson correlation analyses were used to explore relationships between relative delta values that were calculated as changes proportional to the initial value $[\Delta=$ (assessment at follow-up-assessment at baseline)/(assessment at baseline $) \times 100]$ for each cognitive performance domain (T-scores), as well as for demographic, clinical, and pharmacological variables [ $\Delta$ clozapine/NDMC ratio $=$ (clozapine $/ \mathrm{NDMC}$ ratio at follow-up-clozapine/NDMC ratio at baseline)/(clozapine/NDMC ratio at baseline $) \times 100]$. Multiple regression analyses were performed using the delta values of each cognitive score as dependent variables (response, y); demographic (years of education), clinical ( $\triangle$ PANSS total and subscale scores), and pharmacological variables ( $\Delta$ clozapine concentration, $\triangle \mathrm{NDMC}$ concentration, and $\triangle$ clozapine/NDMC ratio) were used as independent variables (predictors, $\mathrm{x}$ ). Each model was built using a stepwise forward-backward variableselection method; the independent variables were entered or removed from the model based on the default p-values, 0.05 and 0.10 , respectively.

\section{RESULTS}

Fifteen patients met the inclusion criteria, including the completion of initial and follow-up assessments. After the initiation of clozapine treatment, initial and follow-up pharmacological assessments were made on days $11.08 \pm 18.77$ [mean \pm standard deviation (SD)] and $106.33 \pm 38.37$, respectively; clinical and cognitive assessments were made on days $3.31 \pm 9.59$ and $104.33 \pm 33.82$, respectively. The demographic, pharmacological, and clinical characteristics of the 15 patients who met the inclusion criteria are provided in Table 1 . The mean patient age was $34.13 \pm 13.31$ years, the proportion of women was $66.7 \%$, and the mean duration of education was $13.47 \pm 2.75$ years. The mean clozapine doses were $135.00 \pm 122.32 \mathrm{mg} / \mathrm{d}$ at the initial assessment and $199.17 \pm 86.42 \mathrm{mg} / \mathrm{d}$ at the follow-up assessment. The total PANSS scores were $77.92 \pm 19.70$ at the initial assessment and $75.27 \pm 18.27$ at the follow-up assessment. Compared with the initial assessment, the NDMC concentration was significantly elevated at the follow-up assessment $(\mathrm{z}=-1.988, \mathrm{p}=0.047)$; however, the clozapine dosage $(\mathrm{t}=-1.850$, $\mathrm{df}=14, \mathrm{p}=0.086)$, clozapine concentration $(\mathrm{t}=-0.961, \mathrm{df}=14, \mathrm{p}=$ $0.353)$, clozapine/NDMC ratio $(\mathrm{t}=-0.180, \mathrm{df}=14, \mathrm{p}=0.860)$, PANSS total scores $(\mathrm{z}=-0.035, \mathrm{p}=0.972)$, and PANSS subscale scores (positive: $\mathrm{t}=1.156, \mathrm{df}=14, \mathrm{p}=0.270$; negative: $\mathrm{t}=0.047$, $\mathrm{df}=14, \mathrm{p}=0.963$; general: $\mathrm{z}=-0.236, \mathrm{p}=0.814$ ) did not significantly change.

Cognitive outcomes are presented as T-scores in Table 2. Processing speed demonstrated a significant elevation in Tscore at the follow-up assessment, compared to the initial assessment $(\mathrm{t}=-2.229, \mathrm{df}=14, \mathrm{p}=0.043)$; however, no significant individual changes were observed in its three corresponding tests [TMT ( $\mathrm{t}=-1.605, \mathrm{df}=14, \mathrm{p}=0.131)$, BACS $(\mathrm{t}=-1.493, \mathrm{df}=14$, $\mathrm{p}=0.158)$, and Fluency $(\mathrm{t}=-1.405, \mathrm{df}=14, \mathrm{p}=0.182)]$. Attention/ vigilance and its corresponding test, CPT-IP, showed a significant elevation in T-score at the follow-up assessment, compared to the initial assessment $(\mathrm{t}=-3.031, \mathrm{df}=14, \mathrm{p}=0.009)$. Although there was no significant change in the working memory domain $(\mathrm{t}=-1.519, \mathrm{df}=14, \mathrm{p}=0.151)$, there was a significant elevation in T-score in the letter-number span ( $\mathrm{t}=-2.359, \mathrm{df}=14$, $\mathrm{p}=0.033$ ) at the follow-up assessment, compared with the initial assessment; notably, there was no change in the spatial span $(\mathrm{t}=-0.922, \mathrm{df}=14, \mathrm{p}=0.372)$. The overall composite score also 
exhibited a significant enhancement at the follow-up assessment, compared to the initial assessment $(\mathrm{t}=-2.275, \mathrm{df}=14, \mathrm{p}=$ 0.040). The remaining domains [i.e., verbal learning $(t=0.372$, $\mathrm{df}=14, \mathrm{p}=0.715)$, visual learning $(\mathrm{t}=-0.801, \mathrm{df}=14, \mathrm{p}=0.437)$, reasoning and problem solving $(\mathrm{t}=-0.994, \mathrm{df}=14, \mathrm{p}=0.337)$, and social cognition $(\mathrm{t}=0.294, \mathrm{df}=14, \mathrm{p}=0.773)]$ exhibited no changes between initial and follow-up assessments.

Correlations between the $\triangle$ clozapine/NDMC ratio and delta values of each cognitive domain are presented in Figure 2. The values of 4 patients that had initial assessments before clozapine initiation were not included in the analysis. $\Delta$ Attention/ vigilance $(\mathrm{r}=-0.678, \mathrm{p}=0.022), \Delta$ social cognition $(\mathrm{r}=-0.646$,

Table 1. Demographic, pharmacologic, and clinical characteristics of 15 patients with schizophrenia who received clozapine treatment

\begin{tabular}{|c|c|c|c|c|c|c|c|}
\hline \multirow{2}{*}{ Parameters } & \multicolumn{2}{|c|}{ Initial assessment } & \multicolumn{2}{|c|}{ Follow-up assessment } & \multirow{2}{*}{$\mathrm{t}^{\mathrm{a}}$} & \multirow{2}{*}{$Z^{b}$} & \multirow{2}{*}{$\mathrm{p}$-value } \\
\hline & Mean & $\mathrm{SD}$ & Mean & $\mathrm{SD}$ & & & \\
\hline $\operatorname{Sex}(M / F)$ & $5 / 10$ & & & & & & \\
\hline Age (y) & 34.13 & 13.31 & & & & & \\
\hline Education (y) & 13.47 & 2.75 & & & & & \\
\hline Clozapine dosage (mg/d) & 135.00 & 122.32 & 199.17 & 86.42 & -1.850 & & 0.086 \\
\hline Clozapine concentration $(\mathrm{nmol} / \mathrm{L})$ & 260.62 & 235.42 & 348.91 & 225.21 & -0.961 & & 0.353 \\
\hline NDMC concentration (nmol/L) & 133.86 & 139.70 & 222.68 & 141.54 & & -1.988 & $0.047^{*}$ \\
\hline Clozapine/NDMC ratio & $1.68^{c}$ & $1.45^{\mathrm{c}}$ & 1.74 & 0.70 & -0.180 & & 0.860 \\
\hline \multicolumn{8}{|l|}{ Positive and Negative Syndrome Scale } \\
\hline Total score & 77.92 & 19.70 & 75.27 & 18.27 & & -0.035 & 0.972 \\
\hline Positive score & 17.38 & 6.07 & 15.00 & 4.63 & 1.156 & & 0.270 \\
\hline Negative score & 20.69 & 5.43 & 20.00 & 5.48 & 0.047 & & 0.963 \\
\hline General score & 39.85 & 10.44 & 40.27 & 10.41 & & -0.236 & 0.814 \\
\hline
\end{tabular}

${ }^{a}$ t-value of paired t-test, ${ }^{b} \mathrm{Z}$-value of Wilcoxon signed-rank test, ${ }^{\mathrm{c}}$ The values of 4 patients that had initial assessments before clozapine initiation were not included in the analysis. ${ }^{*} \mathrm{p}<0.05$. NDMC: $\mathrm{N}$-desmethylclozapine

Table 2. Standardized T-scores of cognitive tests at initial and follow-up assessments

\begin{tabular}{|c|c|c|c|c|c|c|}
\hline \multirow{2}{*}{ MATRICS consensus cognitive battery } & \multicolumn{2}{|c|}{ Initial assessment } & \multicolumn{2}{|c|}{ Follow-up assessment } & \multirow{2}{*}{$\mathrm{t}$} & \multirow{2}{*}{ p-value } \\
\hline & Mean & SD & Mean & $\mathrm{SD}$ & & \\
\hline Processing speed & 27.87 & 14.35 & 34.00 & 18.13 & -2.229 & $0.043^{*}$ \\
\hline Trail Making Test, part A (TMT) & 33.47 & 12.95 & 39.87 & 20.72 & -1.605 & 0.131 \\
\hline Symbol coding task (BACS) & 31.27 & 11.28 & 34.67 & 14.23 & -1.493 & 0.158 \\
\hline Category fluency (Fluency) & 36.53 & 8.94 & 39.40 & 12.06 & -1.405 & 0.182 \\
\hline Attention/vigilance & 31.27 & 13.38 & 38.13 & 14.47 & -3.031 & $0.009^{* *}$ \\
\hline Continuous Performance Test, Identical Pairs (CPT-IP) & 31.27 & 13.38 & 38.13 & 14.47 & -3.031 & $0.009^{* *}$ \\
\hline Working memory & 31.87 & 8.91 & 35.33 & 10.89 & -1.519 & 0.151 \\
\hline Spatial Span test (SS) & 42.33 & 7.90 & 45.07 & 12.25 & -0.922 & 0.372 \\
\hline Letter-Number Span test (LNS) & 27.20 & 8.26 & 30.87 & 7.97 & -2.359 & $0.033^{*}$ \\
\hline Verbal learning & 32.73 & 8.47 & 32.20 & 6.31 & 0.372 & 0.715 \\
\hline Hopkins Verbal Learning Test-Revised (HVLT-R) & 32.73 & 8.47 & 32.20 & 6.31 & 0.372 & 0.715 \\
\hline Visual learning & 34.27 & 15.25 & 36.53 & 10.75 & -0.801 & 0.437 \\
\hline Brief Visuospatial Memory Test-Revised (BVMT-R) & 34.27 & 15.25 & 36.53 & 10.75 & -0.801 & 0.437 \\
\hline Reasoning and problem solving & 38.13 & 11.07 & 40.67 & 8.47 & -0.994 & 0.337 \\
\hline Mazes test (Mazes) & 38.13 & 11.07 & 40.67 & 8.47 & -0.994 & 0.337 \\
\hline Social cognition & 31.53 & 7.69 & 30.86 & 12.97 & 0.294 & 0.773 \\
\hline Managing Emotions test (ME) & 31.53 & 7.69 & 30.86 & 12.97 & 0.294 & 0.773 \\
\hline Overall composite score & 21.60 & 10.36 & 28.64 & 12.25 & -2.275 & $0.040^{*}$ \\
\hline
\end{tabular}

${ }^{*} \mathrm{p}<0.05,{ }^{* *} \mathrm{p}<0.01$. MATRICS: Measurement and Treatment Research to Improve Cognition in Schizophrenia, BACS: Brief Assessment of Cognition in Schizophrenia 
$\mathrm{p}=0.032)$, and $\Delta$ composite score $(\mathrm{r}=-0.610, \mathrm{p}=0.046)$ were significantly correlated with the $\triangle$ clozapine/NDMC ratio; whereas other domains [e.g., $\Delta$ processing speed $(\mathrm{r}=-0.366, \mathrm{p}=0.268)$, $\Delta$ working memory $(\mathrm{r}=-0.561, \mathrm{p}=0.072), \Delta$ verbal learning $(\mathrm{r}=$ -0.370, $\mathrm{p}=0.263), \Delta$ visual learning $(\mathrm{r}=-0.489, \mathrm{p}=0.127)$, and $\Delta$ reasoning and problem solving $(\mathrm{r}=-0.412, \mathrm{p}=0.208)]$, showed no correlations (Figure 2). $\Delta$ Working memory and $\Delta$ social cognition were significantly correlated with other variables: $\Delta$ Working memory was correlated with the $\Delta$ clozapine concentration $(\mathrm{r}=-0.689, \mathrm{p}=0.019)$ and the $\triangle$ NDMC concentration $(\mathrm{r}=-0.689, \mathrm{p}=0.019)$, while $\Delta$ social cognition was associ- ation with the $\Delta$ clozapine concentration ( $\mathrm{r}=-0.762, \mathrm{p}=0.006)$.

The results of multiple regression analysis, which examined delta values of cognitive domains as dependent variables, are summarized in Table 3 . The values of 4 patients that had initial assessments before clozapine initiation were not included in the analysis. $\Delta$ Attention/vigilance was negatively associated with the $\Delta$ clozapine/NDMC ratio $(\mathrm{B}=-1.083, \mathrm{SE} \mathrm{B}=0.418, \beta=$ $-0.676, \mathrm{p}=0.032$ ), whereas other $\Delta$ cognitive domain scores displayed no association with this ratio. $\Delta$ Working memory and $\Delta$ social cognition were negatively associated with the $\Delta$ clozapine concentration ( $\Delta$ working memory: $\mathrm{B}=-0.384, \mathrm{SE} B=0.141, \beta=$

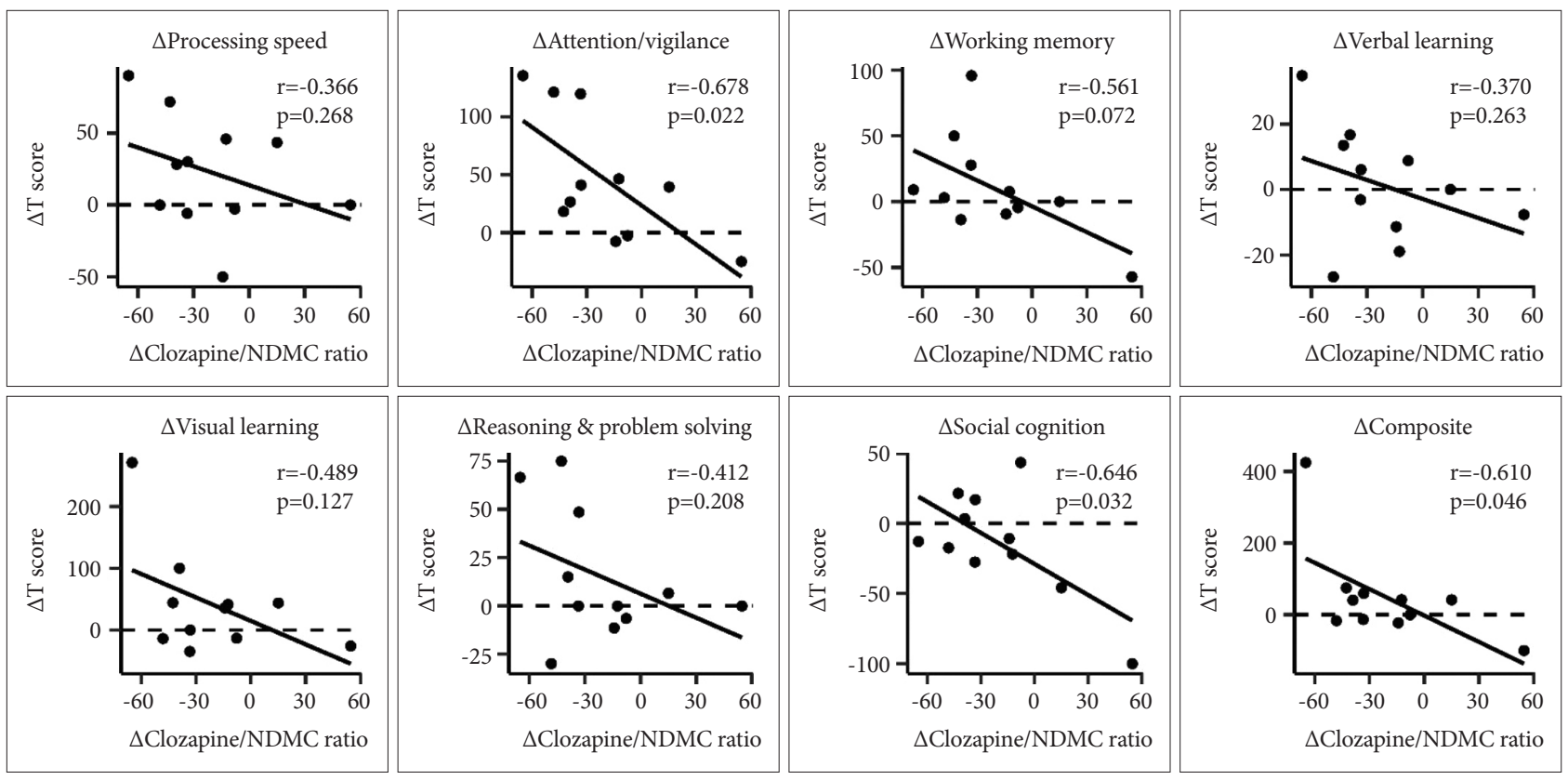

Figure 2. Scatter plots of the $\Delta$ clozapine/NDMC ratio and $\Delta$ standardized T-scores for performance in cognitive domains. The Pearson correlation coefficient $r$ and $p$-value of each cognitive domain are shown in each panel. The values of 4 patients that had initial assessments before clozapine initiation were not included in the analysis. $\Delta=$ (assessment at follow up - assessment at baseline)/(assessment at baseline $) \times 100, \Delta$ clozapine/NDMC ratio=(clozapine/NDMC ratio at follow-up - clozapine/NDMC ratio at baseline)/(clozapine/NDMC ratio at baseline) $\times 100$. NDMC: N-desmethylclozapine.

Table 3. Results of stepwise multiple regression analyses with delta values of all cognitive domains and their corresponding tests as dependent variables

\begin{tabular}{|c|c|c|c|c|}
\hline Model & B & SE B & $\beta$ & p-value \\
\hline \multicolumn{5}{|c|}{$\Delta$ Attention/vigilance $\left(\mathrm{R}^{2}=0.457, \mathrm{p}=0.032^{*}\right)$} \\
\hline (constant) & 27.920 & 16.555 & & 0.130 \\
\hline$\Delta$ Clozapine/NDMC ratio & -1.083 & 0.418 & -0.676 & $0.032^{*}$ \\
\hline \multicolumn{5}{|c|}{$\Delta$ Working memory $\left(\mathrm{R}^{2}=0.483, \mathrm{p}=0.026^{*}\right)$} \\
\hline (constant) & 8.744 & 9.847 & & 0.400 \\
\hline$\Delta$ Clozapine concentration & -0.384 & 0.141 & -0.695 & $0.026^{*}$ \\
\hline \multicolumn{5}{|c|}{$\Delta$ Social cognition $\left(\mathrm{R}^{2}=0.769, \mathrm{p}=0.001^{* *}\right)$} \\
\hline (constant) & -22.206 & 5.643 & & $0.004^{* *}$ \\
\hline$\Delta$ Clozapine concentration & -0.416 & 0.081 & -0.877 & $0.001^{* *}$ \\
\hline
\end{tabular}

The values of 4 patients that had initial assessments before clozapine initiation were not included in the analysis. $\Delta=$ (assessment at follow up assessment at baseline $) /($ assessment at baseline $) \times 100, \Delta$ clozapine/NDMC ratio=(clozapine/NDMC ratio at follow-up-clozapine/NDMC ratio at baseline $) /($ clozapine/NDMC ratio at baseline $) \times 100 .{ }^{*} \mathrm{p}<0.05,{ }^{* *} \mathrm{p}<0.01$. B: parameter estimate, SE B: standard error of B, $\beta$ : standardized B, NDMC: N-desmethylclozapine 
-0.695, $\mathrm{p}=0.026 ; \Delta$ social cognition: $\mathrm{B}=-0.416, \mathrm{SE} \mathrm{B}=0.081, \beta=$ $-0.877, \mathrm{p}=0.001)$. $\Delta$ Processing speed, $\Delta$ verbal learning, $\Delta$ visual learning, $\Delta$ reasoning and problem solving, and $\Delta$ composite score in the cognitive domains exhibited no significant associations with any independent variables.

\section{DISCUSSION}

To the best of our knowledge, this is the first study to examine the relationship between the $\triangle$ clozapine/NDMC ratio and cognitive performance in patients with schizophrenia at the initiation of clozapine treatment. The $\triangle$ clozapine/NDMC ratio was strongly and negatively associated with improvements in attention/vigilance performance; these findings suggested that NDMC (a potent partial agonist of muscarinic M1, M3, and $\mathrm{M} 5$ receptors) can contribute to cognitive enhancement in patients with schizophrenia who receive clozapine treatment. Notably, this association was independent of symptom severity.

Our findings extend those of previous cross-sectional studies regarding the relationship between this ratio and cognitive performance in patients with schizophrenia who receive clozapine treatment, which have reported that lower clozapine/ NDMC ratios were associated with higher cognitive performance. ${ }^{14,20-23}$ Because of the differing actions of clozapine and NDMC on the cholinergic system, the clozapine/NDMC concentration ratio (rather than clozapine dose or either concentration alone) has been proposed as a predictor of cognitive performance in patients undergoing clozapine treatment. ${ }^{20}$ However, the cross-sectional design of the prior study only allowed examination of correlations between the clozapine/ NDMC ratio and cognitive performance; neither a causal relationship between the change in cognitive performance and the clozapine/NDMC ratio nor the impact of changing that ratio on cognitive performance could be demonstrated. Analysis of pharmacological and cognitive assessments at two time points enabled examination of the relationship between the $\Delta$ clozapine/NDMC ratio and cognitive performance.

To our knowledge, only one previous study used a longitudinal approach to assess the association between the clozapine/ NDMC ratio and cognition. ${ }^{24}$ That study, which was notable for exploring changes in cognition as a function of changes in pharmacologic factors, revealed a negative correlation between the clozapine/NDMC ratio and cognition. However, that study assessed patients with unknown duration of clozapine treatment, used simplified versions of clinical and cognitive assessments (i.e., the positive sub-score of the Clinical Global Impression for Schizophrenia and the BACS), did not analyze the scores of subtests of the BACS due to its limited sample size, and measured changes in pharmacological and cognitive variables directly instead of changes proportional to the initial value; thus, it was difficult to determine whether the analysis was appropriately powered to evaluate this association. ${ }^{24}$

Our study analyzed the data of patients with schizophrenia who were starting clozapine treatment and who had their initial assessment within one month from the initiation of clozapine treatment and a follow-up assessment at more than 2 months after initiation of clozapine. Although a cross-sectional study should utilize a time point that represents a stable status for measurement of pharmacologic and cognitive variables, we presumed that analysis of patients who were starting clozapine treatment (rather than patients with long histories of clozapine treatment) would highlight the effects of the $\Delta$ clozapine/NDMC ratio on cognitive performance. Furthermore, for precise assessment of clinical and cognitive statuses, we used a validated instrument and its cognitive sub-domains, along with a delta value proportional to its initial value. MCCB, a cognitive battery comprising assessments of seven cognitive domains that was used in the present study, is considered the FDA gold standard outcome measure of cognitive treatment effects in clinical trials involving patients with schizophrenia, ${ }^{27,28}$ the PANSS is a frequently used scale with three subscales that separately assess positive, negative, and general symptoms. ${ }^{26}$

The $\Delta$ clozapine/NDMC ratio had a significant impact on attention/vigilance performance. Lower clozapine/NDMC ratios are associated with greater cholinergic agonism, which mediates cognitive functioning and influences the performance of tasks that heavily depend on complex attentional processes by modulation of attention and encoding memory. ${ }^{21,30}$ This association was examined as early as within 2-4 months after initiation of clozapine treatment; it was independent of changes in the severities of positive, negative, general, and total symptoms. Although $\Delta$ social cognition and $\Delta$ composite score were correlated with the $\Delta$ clozapine/NDMC ratio, these associations did not persist during multiple regression analysis. Identification of influencing factors and mechanisms of changes in other cognitive domains require further analyses.

Attention/vigilance, processing speed, aspects of the working memory domain, and the overall composite score showed elevated T-scores at the follow-up assessment, compared to the initial assessment. Notably, the stepwise multiple regression model revealed that $\Delta$ attention/vigilance had causal relations with the $\Delta$ clozapine/NDMC ratio, and that $\Delta$ working memory and $\Delta$ social cognition displayed causal relations with the $\Delta$ clozapine concentration, whereas other $\Delta$ cognitive domains did not exhibit significant associations with any independent variables. Most other studies either reported no significant associations between clozapine concentration and cognition, ${ }^{20,22,23}$ or have not reported the results of association analysis with clozapine concentration; ${ }^{21}$ one study reported a significant correlation between the $\triangle$ clozapine concentration and the $\triangle \mathrm{BACS}$ 
composite score. ${ }^{24}$ Consistent with the findings of a previous publication, ${ }^{24}$ our study revealed that $\Delta$ working memory and $\Delta$ social cognition were causally related to the $\Delta$ clozapine concentration. Most studies mentioned previously were crosssectional in nature, whereas the study that reported a significant correlation between the $\Delta$ clozapine concentration and $\Delta$ cognitive performance was designed as a longitudinal study. This suggests that the differences among studies in terms of the correlation with clozapine concentration might be due to different study designs; the longitudinal study examined delta values, whereas the cross-sectional studies used values from a single time point.

Our study assessed the impacts of changes in the clozapine/ NDMC ratio on cognitive performance; however, the change in that ratio was not controlled by the researchers, but occurred naturally depending on the rate at which each patient metabolized clozapine. ${ }^{31}$ This study confirmed the influence of changes in the clozapine/NDMC ratio on cognitive performance, which suggested that routine monitoring of the clozapine/ NDMC ratio is crucial for prediction of changes in cognition and evaluation of underlying mechanisms; these data could be used to adjust the clozapine dose to control the ratio. The results of this study imply that artificial reduction of the ratio by manipulation of factors that affect the pharmacokinetics of clozapine might improve cognitive performance in patients with schizophrenia. Targets for manipulation of clozapine pharmacokinetics include hepatic clearance (influenced by polymorphism of CYP1A2 and other related enzymes), ${ }^{32,33}$ presystemic metabolism in the gut, ${ }^{34}$ renal clearance, ${ }^{35}$ and smoking $^{36,37}$ or caffeine intake ${ }^{38}$ statuses. Although future controlled studies with larger sample sizes are required to confirm these results, our study supports recent efforts to target muscarinic receptor neurotransmission and develop an agent that inhibits the metabolism of NDMC alone, thereby enhancing cognition without worsening psychotic symptoms.

When interpreting the results of this study, some limitations should be considered. First, this study used retrospectively collected routine clinical data. Although assessments were routinely performed when patient started clozapine treatment, the timing was not strictly controlled; therefore, few eligible patients satisfied the inclusion criteria. Despite the small sample size, the clozapine/NDMC ratio exhibited a significant association with improvement in attention/vigilance in patients with schizophrenia who were starting clozapine treatment, encouraging us to continue with a well-designed prospective study that could better define the impact of the clozapine/NDMC ratio on improvement of cognitive function in patients with schizophrenia. Second, repeated cognitive assessments might have led to a learning effect that could have influenced the results of follow-up cognitive assessments. Nevertheless, the test- retest reliability of the MCCB is considered excellent, and the learning effects are confirmed to be small. ${ }^{28,29}$ Third, there is a possibility that the improvements of the cognition might be related to the alleviated psychotic symptoms though we put the PANSS score into the statistical analysis as a covariate. Nevertheless, it is unlikely to be the case since there was no significant change in the PANSS score during the study duration. Finally, although there was little or no change in concomitant medications between the initial and follow-up assessments, most patients were taking concomitant medications other than clozapine, which could have had influenced the cholinergic system.

In conclusion, the current findings suggested that clozapine treatment was associated with improvement in cognitive performance, especially in the attention/vigilance domain, in accordance with changes in the clozapine/NDMC ratio in patients with schizophrenia who were starting clozapine treatment. These findings suggested that reduction of the clozapine/NDMC ratio, which would target muscarinic receptor neurotransmission, could enhance cognition in patients with schizophrenia who received clozapine treatment. Our finding also suggested that working memory and social cognition improved in accordance with changes in the clozapine concentration. Future controlled longitudinal studies with larger sample sizes are required to confirm the effects of changes in the clozapine/NDMC ratio or clozapine concentration on cognitive performance.

\section{Acknowledgments}

This work was supported by Research Resettlement Fund for the new faculty of Seoul National University and grant no. 14-2017-007 from the SNUBH Research Fund. The funding source had no role in the study design, data collection, data analysis, data interpretation, or writing of the manuscript.

\section{Conflicts of Interest}

The authors have no potential conflicts of interest to disclose.

\section{Author Contributions}

Conceptualization: all authors. Methodology: all authors. Validation: Euitae Kim. Formal analysis: Royun Park. Investigation: Euitae Kim, Seoyoung Kim. Data curation: Euitae Kim, Royun Park. Writing-original draft preparation: Royun Park. Writing—review \& editing: Euitae Kim, Seoyoung Kim.

\section{ORCID iDs}

Royun Park Seoyoung Kim

Euitae Kim https://orcid.org/0000-0001-5572-4485 https://orcid.org/0000-0001-9709-5461 https://orcid.org/0000-0003-0914-2331

\section{REFERENCES}

1. Owen MJ, Sawa A Mortensen PB. Schizophrenia. Lancet 2016;388:8697.

2. Joyce EM, Roiser JP. Cognitive heterogeneity in schizophrenia. Curr Opin Psychiatry 2007;20:268-272.

3. Danion JM, Huron C, Vidailhet P, Berna F. Functional mechanisms of 
episodic memory impairment in schizophrenia. Can J Psychiatry 2007; 52:693-701.

4. Galderisi S, Rossi A, Rocca P, Bertolino A, Mucci A, Bucci P, et al. Pathways to functional outcome in subjects with schizophrenia living in the community and their unaffected first-degree relatives. Schizophr Res 2016;175:154-160.

5. Citrome L. Unmet needs in the treatment of schizophrenia: new targets to help different symptom domains. J Clin Psychiatry 2014;75(Suppl 1): 21-26.

6. Nielsen RE, Levander S, Kjaersdam Telleus G, Jensen SO, Ostergaard Christensen T, Leucht S. Second-generation antipsychotic effect on cognition in patients with schizophrenia--a meta-analysis of randomized clinical trials. Acta Psychiatr Scand 2015;131:185-196.

7. Davis JM, Chen N, Glick ID. A meta-analysis of the efficacy of secondgeneration antipsychotics. Arch Gen Psychiatry 2003;60:553-564.

8. Leucht S, Cipriani A, Spineli L, Mavridis D, Orey D, Richter F, et al. Comparative efficacy and tolerability of 15 antipsychotic drugs in schizophrenia: a multiple-treatments meta-analysis. Lancet 2013;382: 951-962.

9. Shirazi-Southall S, Rodriguez DE, Nomikos GG. Effects of typical and atypical antipsychotics and receptor selective compounds on acetylcholine efflux in the hippocampus of the rat. Neuropsychopharmacology 2002;26:583-594.

10. Nucifora FC Jr, Mihaljevic M, Lee BJ, Sawa A. Clozapine as a Model for Antipsychotic Development. Neurotherapeutics 2017;14:750-761.

11. Chakos M, Lieberman J, Hoffman E, Bradford D, Sheitman B. Effectiveness of second-generation antipsychotics in patients with treatmentresistant schizophrenia: a review and meta-analysis of randomized trials. Am J Psychiatry 2001;158:518-526.

12. Buchanan RW, Holstein C, Breier A. The comparative efficacy and long-term effect of clozapine treatment on neuropsychological test performance. Biol Psychiatry 1994;36:717-725.

13. Lameh J, Burstein ES, Taylor E, Weiner DM, Vanover KE, Bonhaus DW. Pharmacology of N-desmethylclozapine. Pharmacol Ther 2007; 115:223-231.

14. Weiner DM, Meltzer HY, Veinbergs I, Donohue EM, Spalding TA, Smith TT, et al. The role of M1 muscarinic receptor agonism of N-desmethylclozapine in the unique clinical effects of clozapine. Psychopharmacology (Berl) 2004;177:207-216.

15. Snyder S, Greenberg D, Yamamura HI. Antischizophrenic drugs and brain cholinergic receptors. Affinity for muscarinic sites predicts extrapyramidal effects. Arch Gen Psychiatry 1974;31:58-61.

16. Chew ML, Mulsant BH, Pollock BG, Lehman ME, Greenspan A, Mahmoud RA, et al. Anticholinergic activity of 107 medications commonly used by older adults. J Am Geriatr Soc 2008;56:1333-1341.

17. Davies MA, Compton-Toth BA, Hufeisen SJ, Meltzer HY, Roth BL. The highly efficacious actions of $\mathrm{N}$-desmethylclozapine at muscarinic receptors are unique and not a common property of either typical or atypical antipsychotic drugs: is $\mathrm{M} 1$ agonism a pre-requisite for mimicking clozapine's actions? Psychopharmacology (Berl) 2005;178:451-460.

18. Sur C, Mallorga PJ, Wittmann M, Jacobson MA, Pascarella D, Williams JB, et al. N-desmethylclozapine, an allosteric agonist at muscarinic 1 receptor, potentiates $\mathrm{N}$-methyl-D-aspartate receptor activity. Proc Natl Acad Sci U S A 2003;100:13674-13679.

19. Bondesson U, Lindstrom LH. Determination of clozapine and its Ndemethylated metabolite in plasma by use of gas chromatography-mass spectrometry with single ion detection. Psychopharmacology (Berl) 1988;95:472-475.

20. Rajji TK, Mulsant BH, Davies S, Kalache SM, Tsoutsoulas C, Pollock $\mathrm{BG}$, et al. Prediction of working memory performance in schizophrenia by plasma ratio of clozapine to N-desmethylclozapine. Am J Psychiatry 2015;172:579-585.

21. McArdle PA, De Mel V, DeMonte V, Winckel K, Gore-Jones V, Foley S, et al. An investigation into the relationship between clozapine treat- ment and cognitive performance in patients with treatment resistant schizophrenia. Schizophr Res 2019;206:450-451.

22. Molins C, Carceller-Sindreu M, Navarro H, Carmona C, Pineiro M, Martinez E, et al. Plasma ratio of clozapine to $\mathrm{N}$-desmethylclozapine can predict cognitive performance in treatment-resistant psychotic patients. Psychiatry Res 2017;258:153-157.

23. Rajji TK, Uchida H, Ismail Z, Ng W, Mamo DC, Remington G, et al. Clozapine and global cognition in schizophrenia. J Clin Psychopharmacol 2010;30:431-436.

24. Dal Santo F, Jarratt-Barnham I, González-Blanco L, García-Portilla MP, Bobes J Fernández-Egea E. Longitudinal effects of clozapine concentration and clozapine to $\mathrm{N}$-desmethylclozapine ratio on cognition: a mediation model. Eur Neuropsychopharmacol 2020;33:158-163.

25. Aravagiri M, Marder SR. Simultaneous determination of clozapine and its $\mathrm{N}$-desmethyl and $\mathrm{N}$-oxide metabolites in plasma by liquid chromatography/electrospray tandem mass spectrometry and its application to plasma level monitoring in schizophrenic patients. J Pharm Biomed Anal 2001;26:301-311.

26. Kay SR, Opler LA, Lindenmayer JP. Reliability and validity of the positive and negative syndrome scale for schizophrenics. Psychiatry Res 1988;23:99-110.

27. Kern RS, Gold JM, Dickinson D, Green MF, Nuechterlein KH, Baade LE, et al. The MCCB impairment profile for schizophrenia outpatients: results from the MATRICS psychometric and standardization study. Schizophr Res 2011;126:124-131.

28. Georgiades A, Davis VG, Atkins AS, Khan A, Walker TW, Loebel A, et al. Psychometric characteristics of the MATRICS Consensus Cognitive Battery in a large pooled cohort of stable schizophrenia patients. Schizophr Res 2017;190:172-179.

29. Nuechterlein KH, Green MF, Kern RS, Baade LE, Barch DM, Cohen JD, et al. The MATRICS Consensus Cognitive Battery, part 1: test selection, reliability, and validity. Am J Psychiatry 2008;165:203-213.

30. Hasselmo ME, Sarter M. Modes and models of forebrain cholinergic neuromodulation of cognition. Neuropsychopharmacology 2011;36: 52-73.

31. Couchman L, Morgan PE, Spencer EP, Flanagan RJ. Plasma clozapine, norclozapine, and the clozapine:norclozapine ratio in relation to prescribed dose and other factors: data from a therapeutic drug monitoring service, 1993-2007. Ther Drug Monit 2010;32:438-447.

32. Chetty M, Murray M. CYP-mediated clozapine interactions: how predictable are they? Curr Drug Metab 2007;8:307-313.

33. Urichuk L, Prior TI, Dursun S, Baker G. Metabolism of atypical antipsychotics: involvement of cytochrome p450 enzymes and relevance for drug-drug interactions. Curr Drug Metab 2008;9:410-418.

34. Cheng YF, Lundberg T, Bondesson U, Lindström L, Gabrielsson J. Clinical pharmacokinetics of clozapine in chronic schizophrenic patients. Eur J Clin Pharmacol 1988;34:445-449.

35. Schoretsanitis G, Kane JM, Ruan CJ, Spina E, Hiemke C, de Leon J. A comprehensive review of the clinical utility of and a combined analysis of the clozapine/norclozapine ratio in therapeutic drug monitoring for adult patients. Expert Rev Clin Pharmacol 2019;12:603-621.

36. Rostami-Hodjegan A, Amin AM, Spencer EP, Lennard MS, Tucker GT, Flanagan RJ. Influence of dose, cigarette smoking, age, sex, and metabolic activity on plasma clozapine concentrations: a predictive model and nomograms to aid clozapine dose adjustment and to assess compliance in individual patients. J Clin Psychopharmacol 2004;24: 70-78.

37. Jacob A, Zahr N, Hulot JS, Boussouar S, Vilain J, Lafont-Rapnouil S, et al. Therapeutic drug monitoring of clozapine in a hemodialysed smoking patient with schizophrenia. Ther Drug Monit 2009;31:281-282.

38. Rajkumar AP, Poonkuzhali B, Kuruvilla A, Jacob M, Jacob KS. Clinical predictors of serum clozapine levels in patients with treatment-resistant schizophrenia. Int Clin Psychopharmacol 2013;28:50-56. 